\title{
Simulation of Transmembrane Potential Propagation in Normal and Ischemic Tissue Using Aliev-Panfilov Model
}

\author{
M. Mehdi Seyedebrahimi ${ }^{1 *}$, Yesim Serinagaoglu1,2 \\ ${ }^{1}$ Biomedical Eng. Dep., Middle East Technical University, Ankara, Turkey. \\ 2 Electric and Electronic Eng. Dep., Middle East Technical University, Ankara, Turkey. \\ * Corresponding author. Tel.: +905342376701; email: ebrahimi.mehdi@metu.edu.tr \\ Manuscript submitted July 30, 2016; accepted December 1, 2016. \\ doi: 10.17706/ijbbb.2017.7.1.13-19
}

\begin{abstract}
Mathematical modelling of heart's electrical activity is useful for understanding the details of heart's function, and developing methods for prediction, diagnosis and treatment of various heart diseases. In this work, we modelled the electrical activity of the heart in the three dimensional (3D) ventricular geometry based on transmembrane potential (TMP) distributions. The main intention in this study is to simulate TMP distributions using heart geometry obtained from Magnetic Resonance (MR) Images, and corresponding fiber structure obtained from Diffusion Weighted Images (DWI). Aliev-Panfilov model was used to describe electrical activity of the heart at tissue level, which focuses on the potential wavefront propagation. Using this model, it is also possible to include the anisotropy of the heart muscle in the calculations. Here, we first simulated 3 dimensional mapping of transmembrane distribution and propagation due to an ectopic stimulation on a normal ventricular geometry of the heart. Then, based on action potential morphology changes in a tissue with ischemia, we derived ischemic weight values and equation parameters for our model. By introducing ischemic regions on the ventricular geometry and applying ischemic weight values and parameters, we simulated TMP distribution and propagation in ventricular geometry with partial ischemia.
\end{abstract}

Key words: Transmembrane potential propagation, Aliev-Panfilov model, normal and ischemic tissue, MRI, DWI.

\section{Introduction}

Cardiac arrhythmia refers to any abnormality or perturbation in the normal sequence of electrical impulses. Accordingly, cardiac arrhythmia is the main cause of morbidity and mortality in the developed world. Therefore, understanding mechanism of them is clinically important. Through investigating the underlying mechanism of the cardiac tissue, clinical methods has some disadvantages that make them insufficient for research purposes. These disadvantages are: 1) most of clinical method are invasive which has limited application in clinic 2) some lethal functional status of the heart such as ischemia, and myocardial infarction associated with ischemia, are difficult and sometimes impossible to investigate by clinical studies on patients. However, mathematical modelling with a simplified description of physical phenomena within mathematical description can be used as a good alternative for simulating, treatment, and prediction purposes. Cardiac mathematical models can be mainly divided to two categories; 1) cellular automata, that represents underlying structure by infinite, regular network or finite automaton working at each cell (node) of the network, 2) Reaction-diffusion methods, in which a system of non-linear partial 
differential equations are used to represent the excitation, distribution and propagation in an excitable media [1]. As in this study, we mainly focused on wave front properties rather than dynamics of ionic currents, therefore, we used reaction-diffusion models rather than the cellular models, which require small space and time integration steps and hence the computational cost is extremely high [2]. Trough different reaction-diffusion methods and considering cost, complexity, and obtained information depth as the most important criteria, we used Aliev-Panfilov model, which adequately represents information at cellular level (e.g. Shape of AP and restitution properties) and tissue level (wave front propagation).

Following the results from previous studies [3], the main objective of this study is to introduce an integrated three-dimensional model of the ventricular tissue starting from segmented MR images and related fiber structure (obtained from DW images) and based on transmembrane potential (TMP) distribution. The irregular point cloud of segmented cardiac images and associated fiber structures are then converted to a regular geometry to be appropriate for finite different method that we used to solve Aliev-Panfilov equations. In this work, we firstly focused on focal arrhythmia, called ectopic heart beats, in which beats arise from cells other than the heart's pacemaker cells normally responsible for impulse formation. Furthermore, we simulated TMP propagation on a ventricular geometry with partially ischemic region; ischemia is another heart problem which results in deterioration of electrical and mechanical activity of the heart, and can be observed as action potential (AP) morphology alteration and contraction failure respectively. We based our simulation on AP morphology alteration values during ischemia from experimental and computational studies in the literature [4]-[6].

\section{Methodology}

\subsection{Aliev-Panfilov Model}

Aliev-Panfilov model uses a system of non-linear partial differential equations to describe the excitation and propagation process in excitable media. The model uses cubic polynomial to model excitation and includes recovery variable similar to FHN model. Moreover, the model includes the information of conductivity tensor and refractory period, in which the courses of excitation and recovery can be calculated using equation (1).

$$
\begin{gathered}
\frac{\delta u}{\delta t}=\nabla \cdot(D \nabla u)-k u(u-\alpha(u-1))-u v \\
\frac{\delta v}{\delta t}=\varepsilon(u, v)(-v-k u(u-\alpha-1))
\end{gathered}
$$

where D and $\varepsilon=\varepsilon_{0}+\frac{\mu_{1} \mathrm{v}}{v+\mu_{2}}$ model the conductivity tensor and the refractory period, respectively.Here $\mathrm{u}, \mathrm{v}$, tare dimensionless variables representing the excitation, recovery and time values. $\alpha, \mathrm{k}, \mu_{1}, \mu_{2}$ and $\varepsilon_{0}$ are equation parameters to be determined according to simulated heart function.

\subsection{Fibrous Structure and Heart Geometry}

Three dimensional geometry used in this study is based on MR images of a dog heart (ventricular geometry only) and the corresponding fiber structure obtained from DW images. This geometry was available as part of University of Utah, SCI Institute datasets. The MR images were already segmented as part of the dataset, hence no additional segmentation was applied to the original MR images. The geometry has dimensions of $8.911 \mathrm{~cm}$ along the $\mathrm{x}$ - and z-axes and $11.17 \mathrm{~cm}$ along the $\mathrm{y}$-axis, with in-slice spacing of $1: 00 \mathrm{~mm}$.

In order to make the geometry information appropriate for finite difference calculations, we interpolated the data on congruent cubic meshes. We first inscribed the geometry of the heart in a box with regular 
hexahedral meshes. Each mesh element had $1 \mathrm{~mm}$ length on each side. Afterwards, we defined nodes from the irregular point clouds geometry, which are in the neighborhood (1.5 mm distant) of predefined box points. Finally, we used inverse distance weighting (IDW) interpolation [7] to transfer irregular points of the heart to the box points (interpolation points).

\subsection{Numerical Modelling}

Numerical implementation of the Aliev-Panfilov model expressed in equation (1) is carried out with finite difference method (FDM). A time step of $0.007 \mathrm{~ms}$ was employed for temporal calculations. To discretize the spatial operations,heart geometry is divided into hexahedral (cubic) elements, where potential values are defined at regularly spaced nodes of this discretized domain. Location of each node is denoted by the global indices, $(i, j, k)$, with $i=1,2, \ldots, \mathrm{N}, j=1,2, \ldots, \mathrm{M}, k=1,2, \ldots, \mathrm{K}$, where $\mathrm{N}, \mathrm{M}$ and $\mathrm{K}$ are the number of nodes along the $\mathrm{x}, \mathrm{y}$ and $\mathrm{z}$ directions, respectively. Fig. 1 shows eight of these hexahedral elements, where the center node common to all eight elements is denoted locally as node $l=0$, and all the first neighborhoodnodes of this center node are denoted locally by indices $1=1,2, \ldots, 18$. In this discretized geometry, equation (1) can be approximated as follows:

$$
\begin{gathered}
\frac{\Delta u}{\Delta t}=L(i, j, k)-k u(u-\alpha(u-1))-u v \\
\frac{\Delta v}{\Delta t}=\varepsilon(u, v)(-v-k u(u-\alpha-1))
\end{gathered}
$$

where $L(i, j, k)$ is the discretized form of the Laplace operation, $\nabla .(D \nabla u)$. This discrete function is calculated for every node in the heart geometry. The node of interest is placed as the center node $(l=0)$ in the local eight-element geometry shown in Fig. 1 , and $L(i, j, k)$ is calculated as the weighted sum of the center node potential value and the potential values of its eighteen first order neighborhood nodes as:

$$
L(i, j, k)=\sum_{1}^{18} p_{l} u(l)
$$

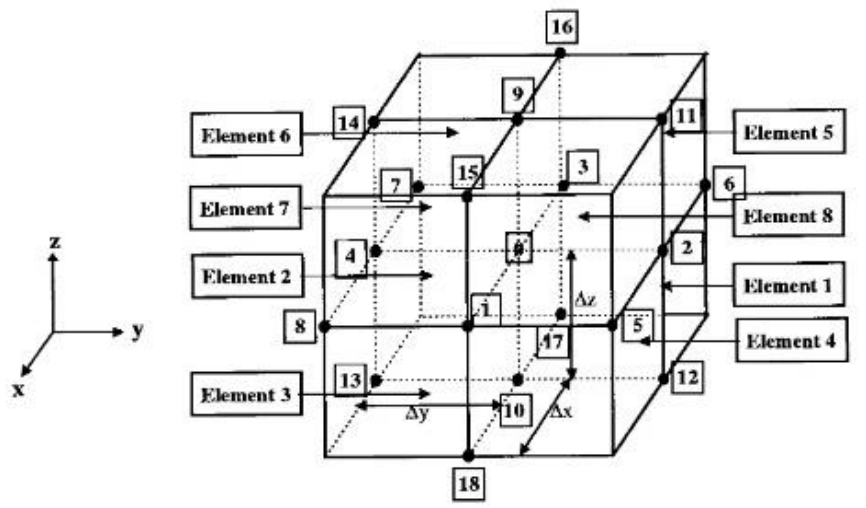

Fig. 1. First neighborhood of each point in heart's geometry [8].

The weight factors $p_{l}$, in equation (3) are calculated by the FDM method as proposed in [8]. This calculation requires the definition of the conductivity tensor $D$ as well at each element. The heartgeometries that we use in this study provide fiber orientations at every node. Using these fiber orientations and the local conductivity values along the fiber direction $(0.16 \mathrm{~S} / \mathrm{m}$ for intracellular and extracellular medium $)$, transverse direction and transmural direction $(0.016 \mathrm{~S} / \mathrm{m}$ for intracellular medium 
and $0.05 \mathrm{~S} / \mathrm{m}$ for extracellular medium in both directions), one can compute the global conductivity tensor at each node according to the conversion described in [9].

\section{Experiments and Results}

In this section, we present TMP distributions and propagation due to an ectopic arrhythmia in the normal and partially ischemic tissue. In the results presented here, the excitation starts at the apex of the heart.

\subsection{Ectopic Heart Beat Propagation on Normal Ventricular Tissue}

Through the ventricles, the action potential duration was $200 \mathrm{msec}$, amplitude was $125 \mathrm{mV}$ and the resting potential is $-85 \mathrm{mV}$. In this simulation, we set the AP amplitude, the resting potential and the AP duration to $125 \mathrm{mV},-85 \mathrm{mV}$ and $200 \mathrm{~ms}$, respectively. The entire TMP propagation takes approximately $311 \mathrm{~ms}$ to span the entire ventricular volume.

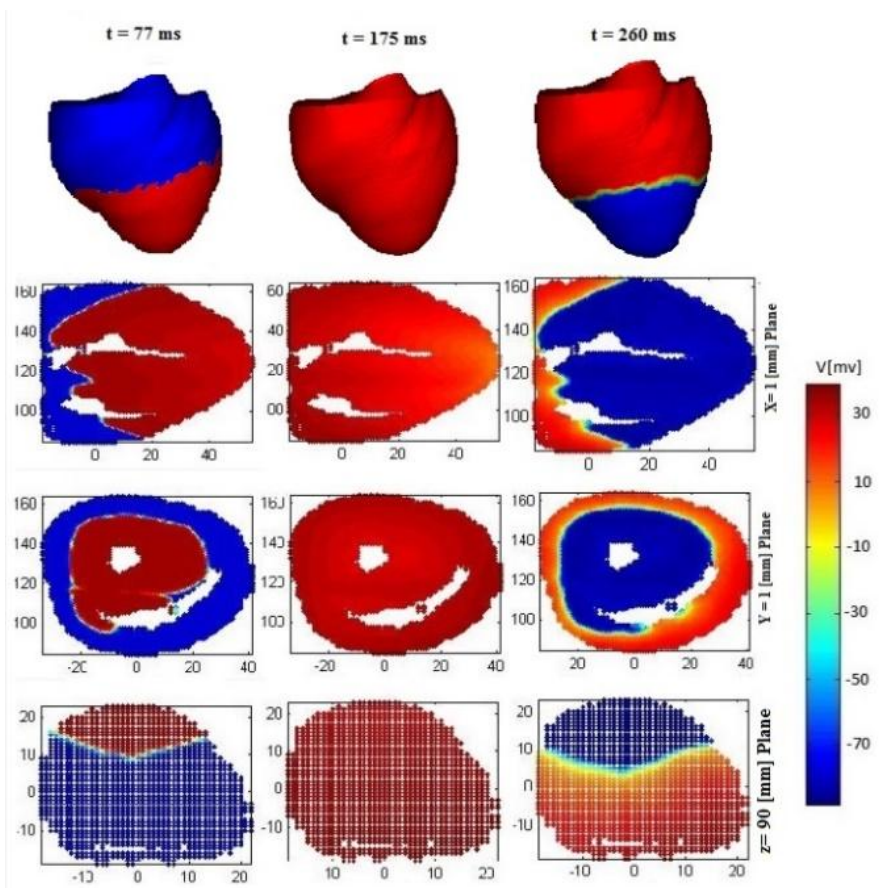

Fig. 2. TMP distribution and propagation for normal tissue due to an ectopic stimulation.

TMP distributions from three different cross-sections $(X=1 \mathrm{~mm}, Y=1 \mathrm{~mm}$ and $\mathrm{Z}=90 \mathrm{~mm})$ are shown in Fig. 2, at three different time instants. As it can be seen from patterns in Fig. 2, at time $77 \mathrm{~ms}$ a wavefront representing depolarization of ventricular myocardium spans the ventricular tissue from the apex to the base. Next, at time $175 \mathrm{~ms}$ almost all of the tissues have been depolarized and are in plateau phase. Finally at time $260 \mathrm{~ms}$, repolarization starts to take place. It is obvious that the same AP characteristics in normal tissue (amplitude, resting potential and duration) are achieved at all locations, as expected from our simulation setup.

\subsection{Simulation of Ectopic Heart Beat in Partially Ischemic Tissue}

According to experimental recordings and investigations [4]-[6], during the first $12 \mathrm{~min}$ of ischemia, resting potential is elevated to $-60 \mathrm{mV}$ from its normal value of $-85 \mathrm{mV}$, action potential duration shortens by almost $50 \%$ and the amplitude decreases to $88 \mathrm{mV}$. In our simulations of TMP's in the ischemic tissue, we aimed to produce AP's similar to the experimental findings in literature. We first introduced a region of ischemia within the ventricular geometry, as shown in Fig. 3. Then, in order to match the experimental APs in the ischemic region, we first calculated new global conductivity tensor values for each point in the 
ischemic region, using intracellular and extracellular local conductivity values of $0.16 \mathrm{~S} / \mathrm{m}$ (longitudinal), $0.018 \mathrm{~S} / \mathrm{m}$ (transverse) and $0,08 \mathrm{~S} / \mathrm{m}$ (longitudinal), $0.03 \mathrm{~S} / \mathrm{m}$ (transverse), respectively. Then, using these new conductivity values, we calculated new weight values for solving equation (3). Finally, using the ischemic weight values and parameters in the ischemic regions and the normal weight values and parameters for the rest of the volume, we simulated TMP distributions and propagation over the ventricles.

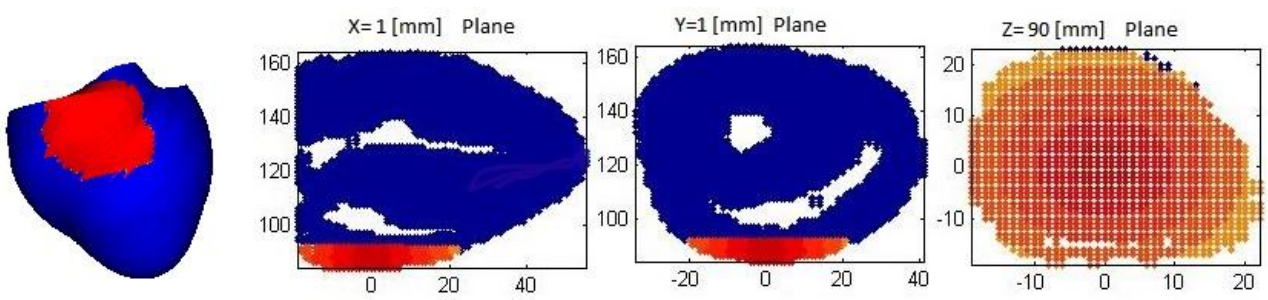

Fig. 3. Ischemic region is shown in reddish color.
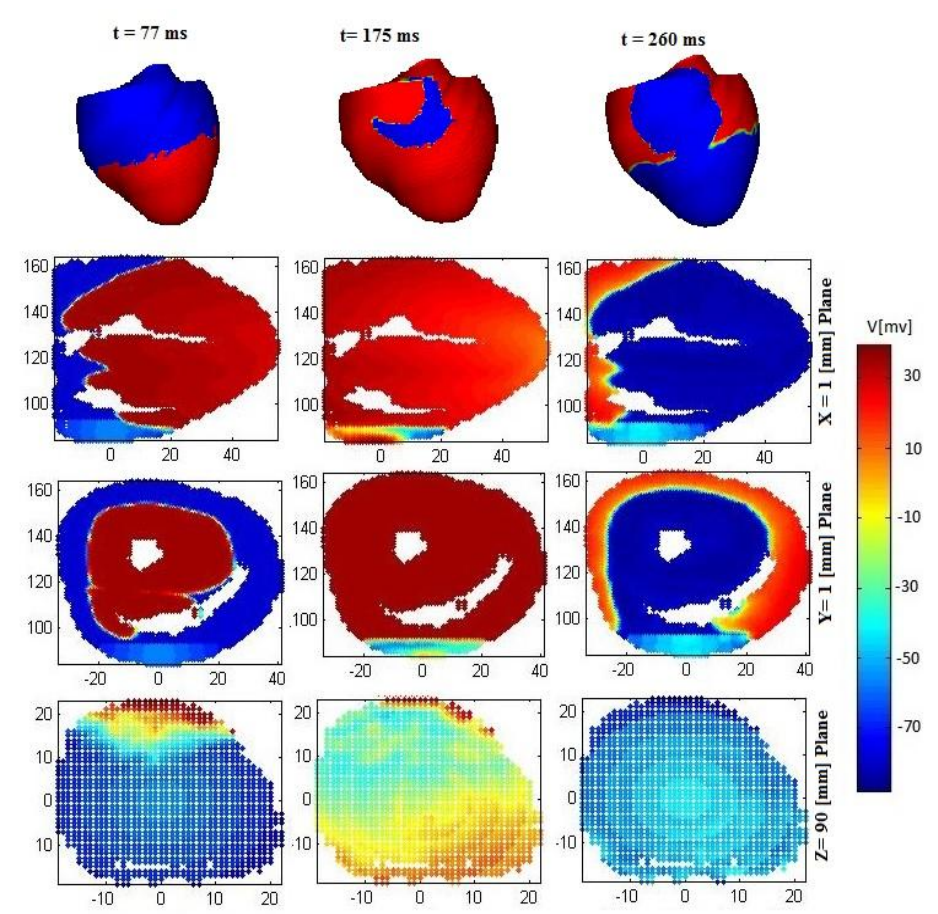

Fig. 4. TMP distribution and propagation for partially ischemic tissue due to an ectopic stimulation.

The result of this simulation, which shows TMP distribution and wavefront propagation, are presented in and Fig. 4 from the same plane cross-sections and at the same sampled times as in Fig. 2. Results from Fig. 2 and 4, it is also observed that the amount of alteration changes gradually from the central part of ischemia to the peripheral region; in a point close to the outer boundary of the ischemic region, where ischemia is considered to be the less severe, the AP waveforms are more similar for the ischemic and the normal cases.

The TMP distributions undergo a disturbance in the ischemic case. This disturbance of pattern is the result of alteration in AP characteristics (amplitude, resting potential and duration), depending on alteration in fiber orientation of the ischemic tissue, initial excitation point and ischemic tissue volume, and degree of ischemia.

\section{Discussion}

In this study, using simplified Aliev-Panfilov cardiac model, we simulated the electrical activity of a dog's heart. The main goal was to be able to obtain a subject specific TMP distribution in the ventricles. As a first 
step to achieve this goal, we started from segmented MR images, and readily available fiber orientations from DW images taken from the SCI Institute datasets. Then from the irregular geometry of the heart, we interpolated the fiber structure to a new regular grid cubic geometry. In this geometry, we calculated the FD weights for any given conductivity tensor definition, and finally we simulated the TMP distributions using the Aliev-Panfilov model that have not physiological meaning. It simulates the overall phenomena adequately and with lower complexity at tissue level and can be used as prediction, diagnostic and treatment tracking tool of heart pathologies. We have carried out our simulations for two cases; normal tissue, and partially ischemic tissue. For both cases, an ectopic stimulation was assumed. In this preliminary work, we can obtain the TMP distributions starting from segmented MR images and the corresponding fiber structure from DW images. In our future work, in order to simulate subject-specific TMP distributions, we will start from the actual medical images and incorporate segmentation as the first step of this work. Another handicap of our study is that in these simulations, we assigned the ischemic region ourselves. However, ischemia does not develop similarly among different patients due to their heart geometry and pathology variations. Another step in our future studies is to determine the region and severity of ischemia also from medical images and obtain more realistic, subject specific ischemic models. Unfortunately, DWI can only provide fiber orientations for isolated hearts; it is not feasible to obtain these images for humans. Thus, we need to rely on generic fiber orientations in our studies, which will introduce errors in our models. The nature of this error should also be a topic for future studies.

\section{Acknowledgment}

This project was supported by the Scientific and Technological Research Council of Turkey (TUBITAK) under grant number 111E258. Magnetic resonance imaging data acquisition project was supported by the National Institute of General Medical Sciences of the National Institutes of Health under grant number P41GM103545 (SCI Institute). The authors would like to thank UgurCunedioglu for his valuable discussions and help with the TMP simulation code.

\section{References}

[1] Sachse, F. B. (2005). Computational Cardiology. Springer.

[2] Aliev, R. R., \& Panfilov, A. V. (1996). A simple two-variable model of cardiac excitation. Chaos, Solitons \& Fractals, 7(3), 293-301.

[3] Cunedioglu, U., \& Yilmaz, B. (2009). Combination of computer simulations and experimental measurements as the training dataset for statistical estimation of epicardial activation maps from venous catheter recordings. IEEE Transactions on Biomedical Engineering, 3(56), 837-845.

[4] Kleber, A. G., Riegger, C. B., \& Janse, M. J. (1987). Extracellular $k^{+}$and $h^{+}$shifts in early ischemia: Mechanisms and relation to changes in impulse propagation. Journal of Molecular and Cellular Cardiology, 19, 35-44.

[5] Weiss, J., \& Shine, K. I. (1982), Extracellular k+ accumulation during myocardial ischemia in isolated rabbit heart. Am J Physiol, 242(4), H619-H628.

[6] Weiss, J. N., Venkatesh, N., \& Lamp, S. T. (1992). Atp-sensitive k+ channels and cellular k+ loss in hypoxic and ischaemic mammalian ventricle. The Journal of Physiology, 447(1), 649-673.

[7] Lu, G. Y., \& Wong, D. W. (2008). An adaptive inverse-distance weighting spatial interpolation technique. Computers \& Geosciences, 34(9), 1044-1055.

[8] Saleheen, H. I., \& Ng, K. T. (1997). New finite difference formulations for general inhomogeneous anisotropic bioelectric problems. IEEE Transactions on Biomedical Engineering, 44(9), 800-809.

[9] Gulrajani, R. M. (1998). Bioelectricity and Biomagnetism. John Wiley and Sons, Inc., New York, NY. Ch. 6. 


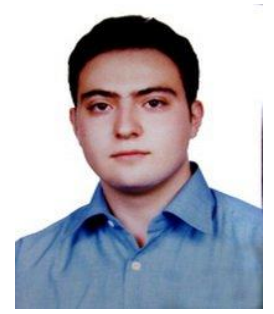

M. Mehdi Seyedebrahimi received the B.S. (2011) degree in electrical and telecommunication engineering from Urmia University, Urmia, Iran, and the M.S. (2014) degree in biomedical engineering from Middle East Technical University, Ankara, Turkey. $\mathrm{He}$ is currently a $\mathrm{PhD}$ candidate of biomedical engineering at Middle East Technical University. His research interests are in statistical and digital signal processing, with particular application to biomedical problems. Specific current interests include forward problems in electrocardiography, modeling the electrical activity of the heart and electrocardiography signal processing.

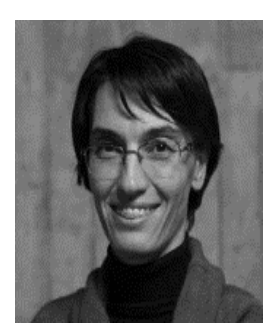

Yesim S. Dogrusoz received the B.S. (1995) and M.S. (1997) degrees in electrical and electronics engineering from Middle East Technical University, Ankara, Turkey, and Ph.D. degree (2003) from Northeastern University, Boston, MA. She is currently an associate professor of electrical and electronics engineering at Middle East Technical University. She is also an affiliated member of the Institute of Applied Mathematics, and Biomedical Engineering Graduate Program at the same university. Her research interests are in statistical and digital signal processing, with particular application to biomedical problems. Specific current interests include forward and inverse problems in electrocardiography, modeling the electrical activity of the heart and electrocardiography signal processing. 